\title{
Comparison of geoeffectiveness of coronal mass ejections and corotating interaction regions
}

\author{
G. Verbanac ${ }^{1}$, S. Živković ${ }^{1}$, B. Vršnak ${ }^{2}$, M. Bandić ${ }^{3}$, and T. Hojsak ${ }^{1}$ \\ ${ }^{1}$ Department of Geophysics, Faculty of Science, University of Zagreb, Horvatovac 95, 10000 Zagreb, Croatia \\ ${ }^{2}$ Hvar Observatory, Faculty of Geodesy, University of Zagreb, Kačićeva 26, 10000 Zagreb, Croatia \\ 3 Preziosa str. 15A, 81927 Muenchen, Germany
}

Received 20 September 2012 / Accepted 6 July 2013

\begin{abstract}
Context. A detailed comparison of the geomagnetic responses to interplanetary coronal mass ejection (ICMEs) and corotating interaction regions (CIRs) during solar cycle 23 was performed using geomagnetic indices $D s t, A p$, and $A E$.

Aims. We aim to find out if there are relative differences in the response of various magnetospheric current systems to the impact of ICMEs and CIRs. In addition, we are exploring the possibility of forecasting geomagnetic activity using the coronagraphic observations of the ICME take-off.

Methods. The peak values of the plasma characteristics of ICMEs and CIRs (velocity $V$, magnetic field $B$, and $B V$ related to the electric field), and geomagnetic indices were investigated by applying the linear and power-law cross correlation analysis. The influence of the time-resolution on the results was performed for two time resolutions obtained by one-hour (three-hour for $A p$ ) and six-hour data averaging.

Results. For ICMEs the power-law fits are found to be important only for the relationships between $B V$ and geomagnetic indices. For $A p$ and $D s t$, there is no difference between the one-hour (three-hour for $A p$ ) and six-hour option. For $A E$, the one-hour data distribution shows more clearly the non-linear dependence on $B V$. Our data set shows that below $B V \sim 5 \mathrm{mV} \mathrm{m}^{-1} \mathrm{ICMEs}$ have practically no geomagnetic effect at low and mid latitudes, but at high latitudes at least some geomagnetic activity will be triggered. For all HSS/CIRs dependencies, a power law is found to better describe the data than the linear fit. The data distributions show that $B V$ has to reach $\sim 4 \mathrm{mV} \mathrm{m}^{-1}$ in order to drive at least some geomagnetic activity at all latitudes. We observed that there are fast CMEs that have almost no geomagnetic effect at low and mid latitudes. On the other hand, at high latitudes, fast CMEs always trigger some geomagnetic activity. This might be have implications for space weather forecasting.

Conclusions. Magnetospheric response to both solar drivers (ICMEs and CIRs) is different at various latitudes, thus results in different development of various current systems within the Earth's magnetosphere and ionosphere. Furthermore, we show that ICMEs and CIRs cause different geomagnetic activity. In the case of ICMEs equatorial current system responses in a linear manner, while the response of the polar-current system is likely to be non-linear. For HSS/CIRs, apparently all current systems respond in a non-linear way, especially the polar current system.
\end{abstract}

Key words. magnetic fields - solar wind - solar-terrestrial relations - Sun: coronal mass ejections (CMEs)

\section{Introduction}

Dynamical processes in the solar atmosphere continuously affect the Earth in a variety of ways. The solar-terrestrial relationship to which we refer in this paper considers phenomena associated with varying outflow of the solar wind that affects the Earth's magnetosphere.

The most important process in the interaction of the solar wind and magnetosphere is magnetic reconnection by which the solar wind magnetic field becomes connected across the dayside magnetopause to the Earth's magnetic field. This enables the energy transfer from the solar wind into the magnetosphere (e.g., Dungey 1961). In particular, energy transfer from the solar wind into the magnetosphere is most favorable when the interplanetary magnetic field (IMF) has a strong southward component $B_{\mathrm{s}}$ (where $B_{\mathrm{s}}=B_{z}$ for $B_{z}<0$ and $B_{\mathrm{s}}=0$ for $B_{z}>0$ ) (e.g., Gonzalez et al. 1994; Kivelson \& Russel 1995). The major solar agents that account for the presence of $B_{\mathrm{s}}$, and thus for the geomagnetic disturbances, are interplanetary coronal mass ejections (ICMEs) and solar wind high speed streams (HSSs) that originate from equatorial coronal holes. For a discussion of the interplanetary causes of geomagnetic storms see, e.g., Tsurutani \& Gonzalez (1997), Zhang et al. (2007b) and Echer et al. (2008).

The magnetic field component $B_{\mathrm{s}}$ associated with ICMEs is contained in their internal structure and/or in the turbulent sheath region between the ICME-driven shock and the ejection (e.g., Lavraud et al. 2006). However, the interaction of the HSS and the slow wind forms a corotating interaction region (CIR), containing regions of compressed plasma (e.g., Gosling 1996, and references therein) indicated by enhanced plasma density and magnetic field intensity. In HSS/CIRs the $B_{\mathrm{S}}$ is associated with these compressions and/or Alfvénic waves in the HSS itself (e.g., Burlaga \& Lepping 1977; Schwenn 1983; Tsurutani \& Gonzalez 1987; Tsurutani et al. 2004; Prolss 2004). The occurrence rate of ICMEs peaks during the solar cycle maximum (e.g., Borovsky \& Denton 2006; Richardson \& Cane 2010, and references therein), and then their geomagnetic effects dominate. On the other hand, during the descending phase of solar cycle the 
geomagnetic activity is dominantly driven by HSS/CIRs, since the CME occurrence rate is low (e.g., Richardson et al. 2001; Richardson \& Cane 2012).

The geoeffectiveness of ICMEs and HSS/CIRs has been studied by many researchers (e.g., Schwenn et al. 2005; Huttunen et al. 2005; Borovsky \& Denton 2006; Georgieva et al. 2006; Lavraud et al. 2006; Alves et al. 2006; Richardson et al. 2006; Yermolaev \& Yermolaev 2006; Vršnak et al. 2007; Zhang et al. 2007b; Gopalswamy 2008; Echer et al. 2008; Verbanac et al. 2011a; Richardson \& Cane 2010, 2012; Verbanac et al. $2011 \mathrm{~b}$, and references therein). In most of these studies, the level of the geomagnetic activity is commonly quantified with the storm-time disturbance index $D s t$, which is a relatively good measure of the ring current strength (Gonzalez \& Tsurutani 1987; Wang et al. 2003; Kane 2005; Alves et al. 2006; Bakare \& Chukwuma 2010). However, we note that consideration of only Dst does not provide the information of the geomagnetic activity at mid and high latitudes. Moreover, the observed $D s t$ has contributions not only from the ring current, but also from magnetopause currents, tail currents, induced currents in the solid Earth, and other sources not yet fully quantified (e.g., Gonzalez et al. 1994; Verbanac et al. 2007).

Verbanac et al. (2011a and b), hereafter Paper I and Paper II, studied the geoeffectiveness of HSS/CIRs during the declining phase of the solar cycle 23, by employing various geomagnetic indices. In this paper we expand the analysis focusing on a detailed comparison of the geomagnetic responses to ICMEs and HSS/CIRs. For this purpose, we separately investigate the ICME- and HSS/CIR-driven geomagnetic activity at low, mid, and high latitudes by analyzing geomagnetic indices $D s t, A p$, and $A E$. The aim is to find out if there are relative differences in the response of different current systems within the Earth's magnetosphere to the impact of ICMEs and HSS/CIRs. Finally, we also relate the coronagraphic observations of the ICME take-off to their in situ properties and the geomagnetic-activity characteristics, looking for a way to forecast the geomagnetic activity at various latitudes using the coronagraphic observations of the ICME take-off.

Following Möstl et al. (2012), we define an ICME as the full interval of the solar wind signature that appears as a change of the background solar wind attributable to the presence of the eruption. In other words, we consider the effect of the overall disturbance, which includes the shock, the sheath region, and the magnetic ejection, making no distinction between the geoeffectiveness of individual structural elements.

This paper is organized as follows. In Sect. 2 we describe the data set and the method of analysis. In Sect. 3 the overall characteristics of the employed samples are presented. Section 4 contains the cross-correlation analysis between ICME parameters and geomagnetic indices ( $D s t, A p$, and $A E$ ), and the analysis of the relationship between the considered geomagnetic indices. The same cross-correlation analysis is preformed for HSS/CIRs in Sect. 5. In Sect. 6 we present the comparison between ICMEs and HSS/CIRs cross-correlation results. In Sect. 7, we relate the ICMEs in situ properties ( $V$ and $B$ ) and geomagnetic indices (Dst, $A p$, and $A E$ ) to the coronagraphic CME speed. Finally, all the results are discussed, and conclusions are drawn in Sect. 8.

\section{Data sets and method}

Our study is based on the following data sets:

- one-hour averages of the solar wind velocity $V$ and the magnetic field strength $B$;
- one-hour averages of geomagnetic indices $D s t$ and $A E$;

- three-hour averages of geomagnetic index $A p$;

- the mean speed of the CME measured by the Large Angle Spectroscopic Coronagraph (LASCO; Brueckner et al. 1995), $V_{\mathrm{CME}}$.

The solar wind data were obtained from the Solar Wind Electron Proton and Alpha Monitor (SWEPAM; McComas et al. 1998) and the Magnetometer (MAG; Smith et al. 1998) onboard the Advanced Composition Explorer (ACE; Stone et al. 1998). We used the merged hour-averaged level- 2 ACE data ${ }^{1}$.

Five of the considered ICMEs have gaps in the ACE velocity data. These data gaps were filled by WIND data when possible. In these cases, the 92-s resolution WIND data ${ }^{2}$ were employed to calculate the hour averages.

Planetary geomagnetic activity index $A p$, the storm-time disturbance index $D s t$, and the auroral electrojet index $A E$ can be downloaded from http://wdc.kugi.kyoto-u.ac. jp/cgi-bin/kp-cgi, http://swdcwww . kugi . kyoto-u . ac.jp/dstdir/, and http://wdc.kugi.kyoto-u.ac.jp/ dstae/index.html, respectively. For more detailed information about the indices, we refer to Prolss (2004) and Verbanac et al. (2011a, 2010). Among the available geomagnetic indices, these three indices have been chosen for the evaluation of the geomagnetic activity since their variations can be physically interpreted and related to the specific current system, for example, the ring current and polar electrojet.

The mean speeds of CMEs in the LASCO field of view are obtained from http: //cdaw.gsfc.nasa.gov/CME-list/.

Although it is known that the southward magnetic field component $B_{\mathrm{s}}$ is the major parameter controlling geomagnetic activity, as the first step in this paper we used the magnetic field strength $B$ for the following reason. We are exploring the possibility that our study will have value for space weather forecasting. Via statistical relationships, we linked the directly observed solar features (e.g., $V_{\mathrm{CME}}$ ) with magnetosheric responses (presented in Sect. 7). The ICME magnetic field strength $B$ and bulk plasma speed $V$ measured in-situ are linked to $V_{\mathrm{CME}}$. On the other hand, we do not know $B_{\mathrm{S}}$ on the Sun and it does not depend on the velocity at the Sun. Prediction of southward $B_{z}$ requires a much more sophisticated approach.

For ICMEs we performed the analysis on two time resolutions, one-hour (three-hour) and six-hour resolutions. The sixhour means are calculated from hourly data of all quantities (for Ap three-hour values). The influence of the time-resolution on the results is presented in Sect. 4.

We focused on six-hour means for the following reasons.

First, when studying the geoeffectiveness (based on the consideration of different geomagnetic indices $D s t, A p$, and $A E$ ) of HSS/CIRs in Paper I, we performed a preliminary analysis testing various options, and found that the six-hour resolution (obtained by averaging one-hour values), is the optimal one for all three indices, since when shorter intervals are applied the results of the correlation analysis do not change much, only the noise level increases. Moreover, we observed prolonged periods of $A E$ activity (discussed in Paper I and Paper II) associated with enhanced convection which provides an additional energy injection in both ring current and polar current. With six-hour resolution such activity is better taken into account. To make a comparison with the magnetospheric response to the impact of ICMEs, we employed the same resolution for the ICME speeds and magnetic fields. Furthermore, since our study aims to be used for the

\footnotetext{
http://www.srl.caltech.edu/ACE/ASC/level2/

2 ftp://spdf.gsfc.nasa.gov/pub/data/wind/swe/ascii
} 
G. Verbanac et al.: Geoeffectiveness of ICMEs and CIRs

Table 1. Overall characteristics of the employed data set (six-hour resolution), compared with characteristics of samples used in some other studies.

\begin{tabular}{|c|c|c|c|c|c|c|c|}
\hline & \multicolumn{7}{|c|}{ ICMEs } \\
\hline & $\begin{array}{c}B \\
(\mathrm{nT}) \\
\end{array}$ & $\begin{array}{c}V \\
\left(\mathrm{~km} \mathrm{~s}^{-1}\right) \\
\end{array}$ & $\begin{array}{c}B V \\
(\mathrm{mV} / \mathrm{m})\end{array}$ & $\begin{array}{l}D s t \\
(\mathrm{nT}) \\
\end{array}$ & $\begin{array}{c}A p \\
\text { (nT) }\end{array}$ & $\begin{array}{c}A E \\
(\mathrm{nT})\end{array}$ & Reference \\
\hline MAX & 48 & 1176 & 45.4 & -341 & 345 & 1575 & \\
\hline MEAN & 21 & 634 & 13.5 & -122 & 115 & 808 & \\
\hline MEDIAN & 18.3 & 611 & 11.4 & $\begin{array}{c}-121 \\
\text { HSS/CIRs }\end{array}$ & 105 & 778 & (63 ICMEs) \\
\hline MAX & 22 & 742 & 12 & -116 & 157 & 874 & \\
\hline MEAN & 14 & 610 & 7 & -52 & 49 & 573 & \\
\hline MEDIAN & 13 & 614 & 6 & -40 & 37 & 596 & (38 HSS/CIRs) \\
\hline
\end{tabular}

purposes of the space weather forecasting, we are interested in how the observed overall interplanetary parameters are related to the overall magnetospheric perturbation. Thus, the longer duration aspect of the solar wind disturbance becomes more important than individual shorter-duration peaks in the data. The duration of the considered events (defined here as the time interval of the enhanced magnetic field) are larger than six hours, so the individual spikes seen at a higher resolution might not represent well the cause of the overall geomagnetic perturbation. We emphasize that by the six-hour averaging we smoothed the one-hour values, but we kept the original information so that the results based on six- and one-hour averages could be compared (Sect. 4)

In the analysis we focus on ICME- and HSS/CIR-related geomagnetic activity during solar cycle 23 .

The ICME sample analyzed in this paper includes 43 events classified as ICME in Table B.1 in Appendix B of Dumbović et al. (2011) which was based on the list of CME-ICME pairs prepared by Schwenn et al. (2005), and 21 CME-ICME pairs from Zhang et al. (2007a). The majority of the selected ICMEdriven storms were driven by a single ICME. Yet, the sample includes several events of a more complex structure resulting from ICME-ICME interactions, which enabled us to encompass also the strongest storms of cycle 23 . We note that this sample represents the general sample of events, which includes different levels of geomagnetic activity, from very low to very high. That allows us to study both the lower and upper limits on the strength of the geomagnetic disturbance.

As in Paper II, here we study the HSS/CIR events that occurred during the ICME-less periods in the declining phase of solar cycle 23: in 2005 (day of year, $D O Y=25-125$ ) and in $2006(D O Y=60-261)$. The original data set contains $32 \mathrm{HSS} / \mathrm{CIR}$ structures (see Paper I and Paper II). We identified four ICMEs just before HSS/CIRs. In these cases it was not possible to distinguish the ICME and HSS/CIR peaks, especially in the magnetic field data. These events were excluded from the analysis. We supplemented this sample by ten HSS/CIRs from Zhang et al. (2007a), out of which only two occurred in 2005, i.e., in the period overlapping with the initial data set. Other events cover different phases of cycle 23 (1998, 2002, 2003, and 2004), when the general state of the heliosphere is much more affected by the CME activity. We note that these events are the strongest CIR-related events.

\section{Overall characteristics of the employed samples}

The 1 AU parameters characterizing the employed samples of HSS/CIRs and ICMEs are considerably different. In Table 1 we present overall characteristics of the samples, where the first three columns describe the 1 AU properties of HSS/CIRs and
ICMEs, whereas the next three columns represent the related geomagnetic activity. For estimated peak values of all considered parameters, we calculated maximum, mean and median values (deWe noted as MAX, MEAN and MEDIAN respectively in Table 1).

The highest $V$ and $B$ peaks found among all the events, are significantly higher for ICMEs than for HSS/CIRs. The differences are also present in average and median values of $B$, whereas the differences are less pronounced for $V$. The differences are also pronounced in the maximum, average and median $B V$ peak values.

The geomagnetic activity related to the analyzed ICMEs, as quantified by all three geomagnetic indices, is significantly stronger than that related to HSS/CIRs. For ICMEs, the highest Dst amplitude $(-341 \mathrm{nT})$ is related with $V=663 \mathrm{~km} \mathrm{~s}^{-1}$, accompanied by strong magnetic field $(B=42 \mathrm{nT})$ The strongest $A p$ peak (345 nT) is associated with the maximum $V$ peak $\left(1176 \mathrm{~km} \mathrm{~s}^{-1}\right)$, accompanied by strong $B(42 \mathrm{nT})$. The highest $A E$ peak $(1575 \mathrm{nT})$ is found for $V=966 \mathrm{~km} \mathrm{~s}^{-1}$ and the strongest $B(48 \mathrm{nT})$.

Following the terminology of Sugiura \& Chapman (1960); Gonzalez et al. (1994), intense storms are those with Dst below $-100 \mathrm{nT}$, moderate storms are those with $D s t$ in the range between $-50 \mathrm{nT}$ and $-100 \mathrm{nT}$, and weak storms fall between $-30 \mathrm{nT}$ and $-50 \mathrm{nT}$. Most of the observed Dst peaks fall into the range $-200<D s t<0 \mathrm{nT}$, and following the above criteria we can say that within our sample of events storms of different intensities occurred, from weak to intense.

For HSS/CIRs, the strongest $D$ st peak $(-116 \mathrm{nT})$ associated and the strongest $A p$ peak $(157 \mathrm{nT})$ are associated with maximum peak $V=742 \mathrm{~km} \mathrm{~s}^{-1}$ and $B=14 \mathrm{nT}$ peak. The highest $A E$ peak (874 nT) is associated with $V=509 \mathrm{~km} \mathrm{~s}^{-1}$ and the $B=17 \mathrm{nT}$.

Most HSS/CIR-driven storms were weak (Dst peaks between $-80 \mathrm{nT}$ and $-20 \mathrm{nT}$ ). These storm levels indicate that intense events have lower probability of occurrence than weaker storms, as already noticed in previous studies (Tsurutani et al. 2006; Alves et al. 2006, see also Paper I and II). Richardson et al. (2006) also show the lower occurrence of weaker events for a 159 CIR sample (see their Fig. 9). We note that, CIRs alone occasionally may produce intense storms (Richardson et al. 2006; Zhang et al. 2003).

We note that maximum peak values of different geomagnetic indices do not occur in the same event. This may indicate that different current systems within the Earth's magnetosphere (as measured by ground magnetometers at different latitudes and quantified by geomagnetic indices) show different responses on the geoeffective solar wind flow. This is observed for both ICMEs and HSS/CIRs. 


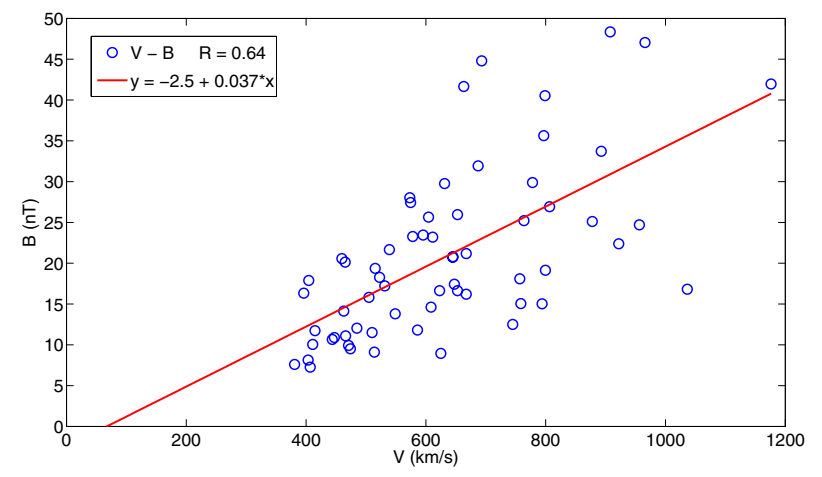

Fig. 1. Relationship between the ICME peak velocity and peak magnetic field. The parameters and the correlation coefficient $R$ of the linear least-squares fit are shown in the inset.

\section{Cross-correlations for ICMEs}

In the following we investigate the relationships between the ICME parameters $B, V$, and $B V$, and geomagnetic indices $A p$, $D s t$, and $A E$, by applying the cross-correlation analysis. For all relationships, we performed linear and power-law least-squares fitting. The latter option is found to be important for the relationships between $B V$ and all three geomagnetic indices, as described below. We employ the simple power-law fit of the form $y=a x^{b}$, which can be solved analytically and does not depend on the used numerical procedure.

The scatter-plot relating the peak values of the ICME magnetic field and velocity is presented in Fig. 1. There is a modest correlation between $B$ and $V(R=0.64)$. The range of values in the data scatter reveals that large values of $B$, for example $B \sim 30 \mathrm{nT}$, occur at $V \sim 600 \mathrm{~km} \mathrm{~s}^{-1}$. However, we note that there are also fast ICMEs without strongly enhanced magnetic fields (e.g., at $V \sim 1050 \mathrm{~km} \mathrm{~s}^{-1}$, according to the scatter plot, $B \sim 15$ nT may be expected). Nevertheless, the fitted linear relation clearly shows a trend that ICMEs with stronger magnetic fields propagate at higher velocities (the fits gives the most probable values i.e., $B=20 \mathrm{nT}$ at $V=600 \mathrm{~km} \mathrm{~s}^{-1}$ and $B=36 \mathrm{nT}$ at $V=1050 \mathrm{~km} \mathrm{~s}^{-1}$ ).

This has implications for geoeffectiveness since the product $B V$, related to the electric field, is enhanced by both factors, $V$ and $B$. Our $V-B$ correlation is generally in agreement with the linear correlations found by other studies (e.g., Gonzalez et al. 1998; Gopalswamy et al. 2008; Dal Lago et al. 2001; Richardson \& Cane 2010). Differences could be attributed to different sample sizes, different ways in which peak-values were determined, and the fact that we did not consider separately different ICMEs substructures (e.g., shock, sheath, MC). For instance, (Richardson \& Cane 2010) obtained modest $V-B$ correlation for magnetic clouds, while there was little correlation for other events. For a discussion of the relationship between ICME magnetic field intensities and speeds see also Owens et al. (2005) and Richardson \& Cane (2010).

In Table 2 we present the linear least squares correlation coefficients between ICME parameters and geomagnetic indices. The highest correlations are for the relationships $B-D s t, B-A p$, and $B V-A p$. The $A E$ is most tightly correlated with $V$ and $B V$ ( $R=0.69$ and $R=0.65$, respectively), whereas $D s t$ is most tightly correlated with $B$ and $B V(R=-0.85$ and -0.79 , respectively). The $D s t$ is generally better correlated with the ICME parameters than $A E$. The only exception is $V-A E$ correlation, which is higher than the $V-D s t$ correlation. The relatively tight correlations between all geomagnetic indices and peak ICME
Table 2. Linear least squares correlation coefficient for the relationship between geomagnetic indices and ICME parameters.

\begin{tabular}{lccc}
\hline \hline & $A p$ & $D s t$ & $A E$ \\
\hline$B$ & 0.83 & -0.85 & 0.60 \\
$V$ & 0.73 & -0.57 & 0.69 \\
$B V$ & 0.82 & -0.79 & 0.65 \\
\hline
\end{tabular}
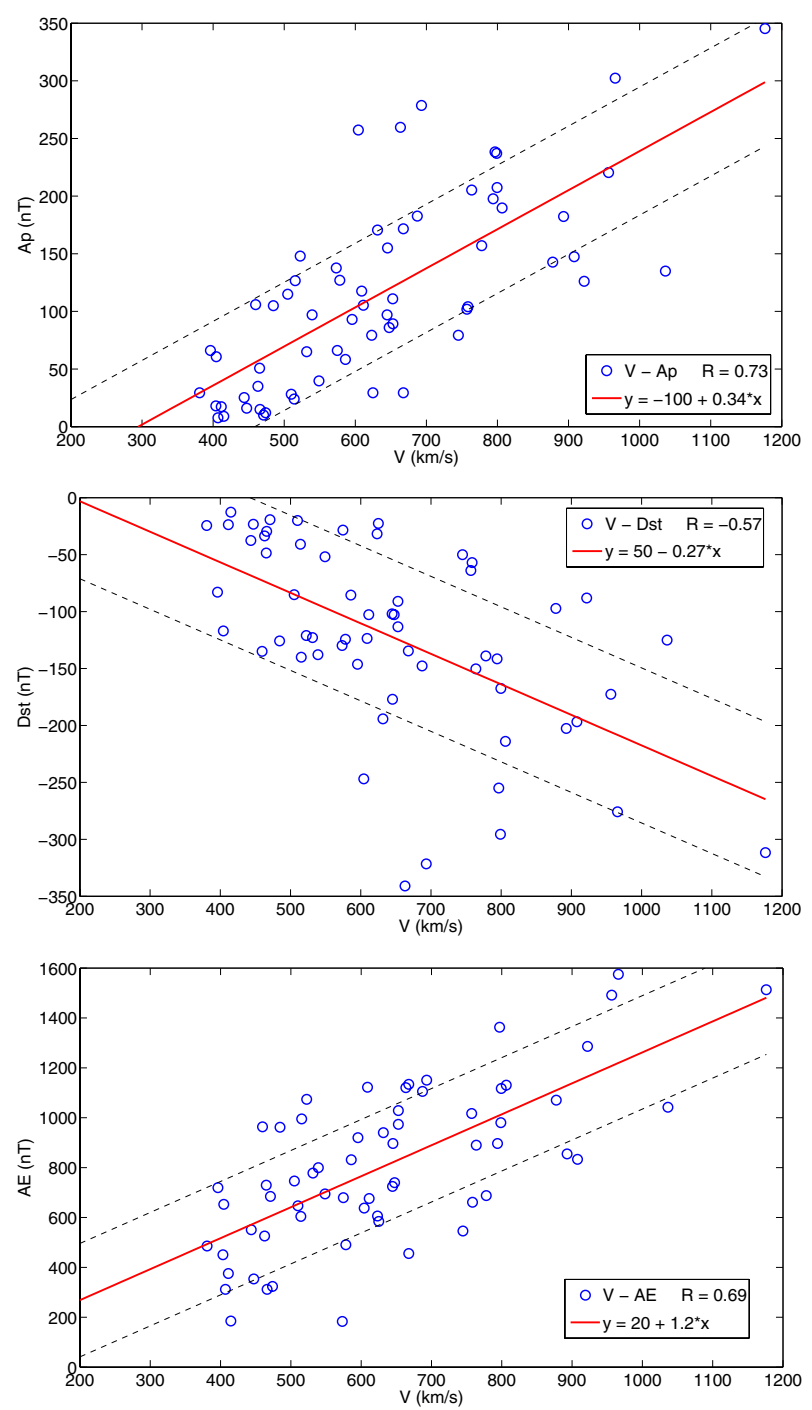

Fig. 2. Correlations $V-A p, V-D s t, V-A E$ for ICMEs. The linear leastsquares fit parameters and the correlation coefficient $R$ are presented in the insets; dashed lines represent the residual standard deviation.

speed may be related to a good $V-B$ relationship $(R=0.64)$, shown in Fig. 1. Both linear relationships $V-D s t$ and $B-D s t$ have also been investigated for example, by Alves et al. (2006) and Gopalswamy et al. (2008). The $V-D$ st relationship is also discussed in e.g., Kane (2005), Richardson \& Cane (2010).

The $V-A p, V-D s t$ and $V-A E$ scatter plots are shown in Fig. 2. In Fig. 3 the scatter plots $B-A p, B-D s t$, and $B-A E$ are displayed. According to the linear regression analysis (Fig. 3) weak, moderate, and intense storms $(-50<D s t<-30 \mathrm{nT}$, $-100<D s t<-50 \mathrm{nT}$, and $D s t<-100$, respectively) are on average caused by ICMEs characterized by, say, $B<15 \mathrm{nT}$, $15<B<20 \mathrm{nT}$, and $B>20 \mathrm{nT}$, respectively. However, the data scatter reveals that these storm levels (weak, moderate, and intense) could also occur for different magnetic field strengths. 

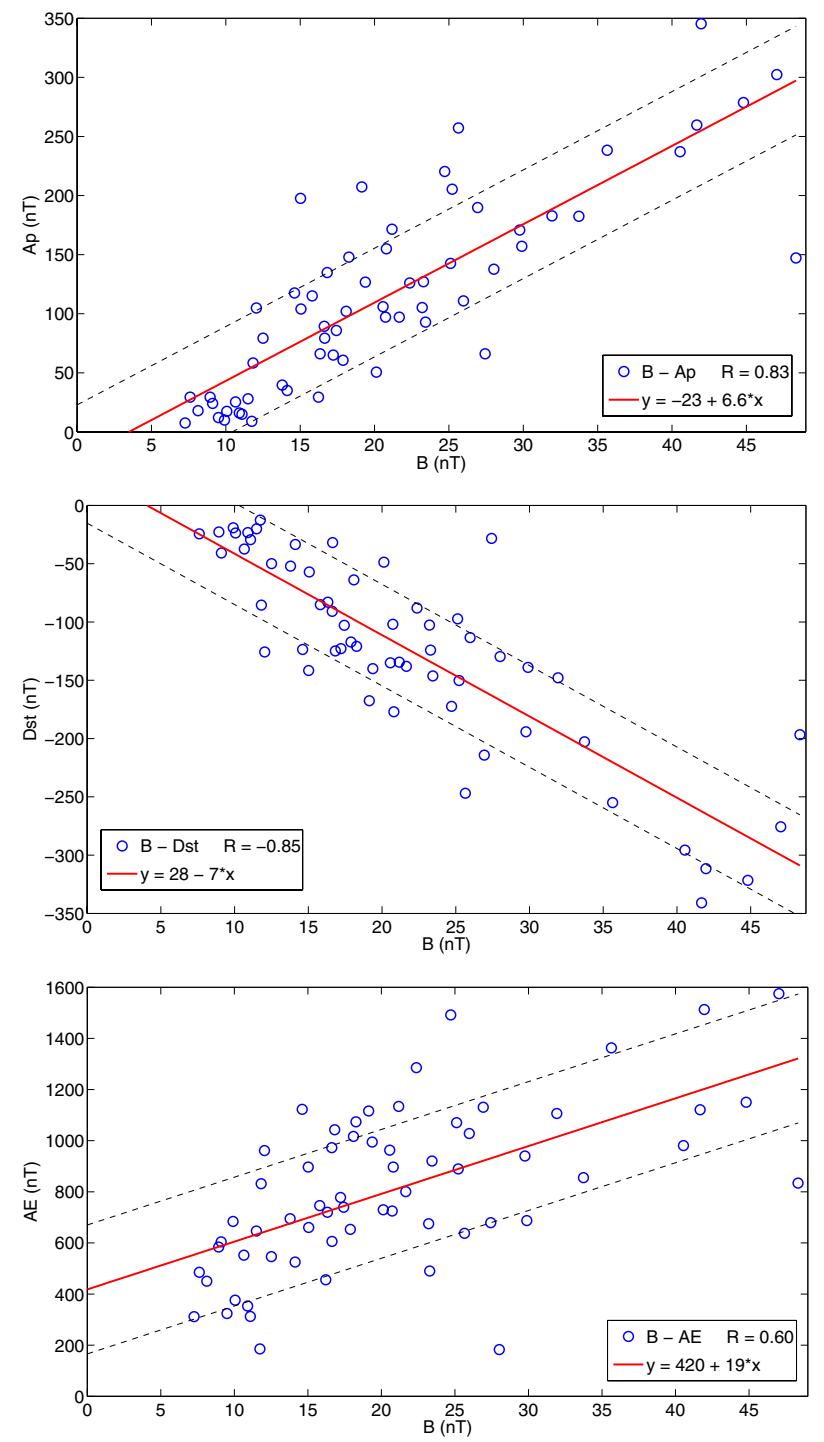

Fig. 3. Correlations $B-A p, B-D s t, B-A E$ for ICMEs. The linear leastsquares fit parameters and the correlation coefficient $R$ are presented in the insets. Dashed lines represent the residual standard deviation.

We note that such values of $B$ are considerably higher than the typical field magnitudes of $5 \mathrm{nT}$ in the quiet solar wind (see, e.g., King 1986).

In Fig. 4 the scatter-plot of $A p, D s t$, and $A E$, versus $B V$ is shown, together with the linear and power-law least-squares fits. These dependencies are investigated for two time resolutions, i.e., applying one-hour (three-hour for $A p$ ) and six-hour averaging (both shown in Fig. 4). Most of the geomagnetic-index peaks are related to $B V$ values below $\sim 35 \mathrm{mV} \mathrm{m}^{-1}$. At six-hour resolution, the highest $A E$ peak ( $1575 \mathrm{nT})$ is found for the strongest $B V$ peak $\left(B V=45 \mathrm{mV} \mathrm{m}^{-1}\right)$. On the other hand, the highest values of $A p$ and of $D s t$ peaks (345 nT and $-341 \mathrm{nT}$, respectively) are associated with a somewhat lower value, $B V=35 \mathrm{mV} \mathrm{m}^{-1}$ and $B V=27 \mathrm{mV} \mathrm{m}^{-1}$, respectively. The data distributions show that, generally, a higher $B V$ causes a stronger geomagnetic activity at all latitudes.

The results of linear fitting $(y=a x+b)$ and power-law fitting $\left(y=a x^{b}\right)$ for different time resolutions are displayed in Table 3 . For both resolutions, the correlation coefficients for the $B V-D s t$ relationship show that linear fit and power-law fit describe the data equally well. We note, the exponent of the power-law fit
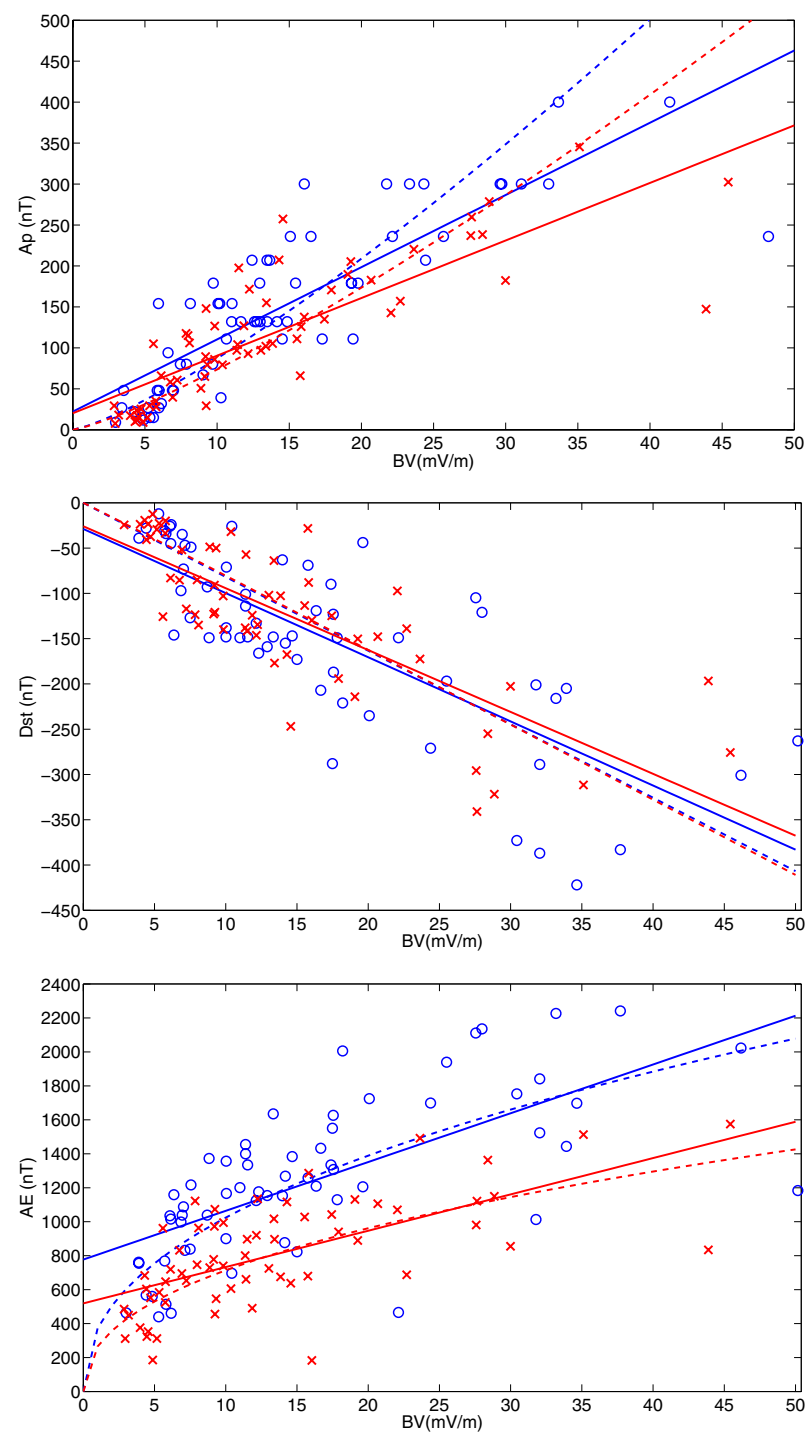

Fig. 4. Linear $y=a x+b$ (solid line) and power-law $y=a x^{b}$ (dashed line) fits for the relationships $B V-A p$ (top), $B V-D s t$ (middle), and $B V-A E$ (bottom) for ICMEs. Blue circles and red crosses represents one-hour resolution data (three-hour resolution for $A p$ ) and six-hour resolution data, respectively. Fit parameters and correlation coefficients are displayed in Table 3.

Table 3. Linear and power-law least-squares fits $\left(y=a x+b, y=a x^{b}\right.$, respectively), and corresponding correlation coefficients $R$, describing the relationships between ICME $B V$ and geomagnetic indices for one/three hour-resolution and six-hour resolution.

\begin{tabular}{lccccccc}
\hline \hline & & $a_{\text {lin }}$ & $b_{\text {lin }}$ & $a_{\text {pow }}$ & $b_{\text {pow }}$ & $R_{\text {lin }}$ & $R_{\text {pow }}$ \\
\hline$D s t$ & $1 \mathrm{~h}$ & 7.1 & 29 & 8.3 & 1 & -0.77 & -0.78 \\
$D s t$ & $6 \mathrm{~h}$ & 6.8 & 26 & 7.8 & 1 & -0.79 & -0.79 \\
\hline$A p$ & $3 \mathrm{~h}$ & 8.8 & 22 & 4.8 & 1.26 & 0.84 & 0.86 \\
$A p$ & $6 \mathrm{~h}$ & 7 & 20 & 4.3 & 1.24 & 0.82 & 0.86 \\
\hline$A E$ & $1 \mathrm{~h}$ & 29 & 780 & 370 & 0.44 & 0.67 & 0.72 \\
$A E$ & $6 \mathrm{~h}$ & 21 & 520 & 270 & 0.43 & 0.65 & 0.63 \\
\hline
\end{tabular}

Notes. Subscripts lin and pow refer to linear and power-law fits, respectively.

at both resolution is close to one, highlighting that $D s t$ is linearly dependent on $B V$ (not depending on the data resolution). The fitted dependencies are shown in the middle panel of Fig. 4. 
Below $\sim 5 \mathrm{mV} \mathrm{m}^{-1}$ all $D$ st peaks are below $-50 \mathrm{nT}$. The fits indicate that, on average, $B V$ has to be around $\sim 10 \mathrm{mV} \mathrm{m}^{-1}$ and $\sim 35 \mathrm{mV} \mathrm{m}^{-1}$ in order to cause an intense geomagnetic storm $(D s t<-100 \mathrm{nT})$ and extreme storm $(D s t<-300 \mathrm{nT})$, respectively. However, the data distribution shows that storms of these intensities could occur at $B V$ even below these values. We note that for very large $B V\left(B V>40 \mathrm{mV} \mathrm{m}^{-1}\right)$ there are storms with $D$ st below -250 nT. However, at such high $B V$ values, we do not have sufficient data points to reach a general conclusion. For additional information about the conditions leading to intense geomagnetic-storm $D$ st levels, we refer to studies by, e.g., Gonzalez \& Tsurutani (1987), Echer et al. (2008), Ji et al. (2010), and Richardson \& Cane (2010).

For the $B V-A p$ relationship, the correlation coefficients show that the power-law dependence is slightly better than the linear dependence at both resolutions (the uppermost panel of Fig. 4). The six-hour resolution data (as well as fitted lines) are moved to somewhat lower values compared to the three-hour resolution data. Below $\sim 5 \mathrm{mV} \mathrm{m}^{-1} A p$ peaks have low values ( $<50 \mathrm{nT}$ ). We note the tendency of a kind of a saturation of $A p$ peak values at $B V>20 \mathrm{mV} \mathrm{m}^{-1}$ which is not well reproduced by the power-law fit.

In the case of the $B V-A E$ relationship the linear and powerlaw fits are very similar at the six-hour resolution, while at the one-hour resolution the power-law provides a better fit. The power-law exponent is close to $1 / 2$, regardless of the resolution. At the six-hour resolution the data (and fits) in the scatter plot are moved toward lower values compared to the data at onehour resolution, as seen in the bottom panel of Fig. 4. It is interesting to note a different type of data distribution compared to $B V-A p$ data. In the case of $A E$, there is a clear upper limit to the data. This upper-limit boundary, located some $500 \mathrm{nT}$ above the power-law fit, rises up to $\sim 25 \mathrm{mV} \mathrm{m}^{-1}$, and then indicates a kind of saturation. On the other hand, at very high $B V$, there is also a lower limit to the data, namely there is no $A E$ below $\sim 600 \mathrm{nT}$. It is also worth noting that even at very low $B V$ peak values ( $\lesssim 5 \mathrm{mV} \mathrm{m}^{-1}$ ), some significant $A E$ values are observed, up to $700 \mathrm{nT}$. We note that the scatter in the $B V$ plots is partly due to the strong field that can be oriented northward (weak activity) or southward (strong activity).

The above results clearly indicate different magnetospheric responses on the same input from the solar wind driver $(B V)$ at various latitudes. Our sample reveals that the equatorial current system's response is linear, while the polar current system's response is likely to be non-linear.

The presented analysis of the influence of the time-resolution influences on the results shows that for $A p$ and $D s t$, there is practically no difference between the one-hour (three-hour for $A p$ ) and six-hour option. For $A E$, the one-hour data distribution shows more clearly the non-linear dependence on $B V$.

The different responses of the polar and equatorial magnetosphere reflect the different nature of geomagnetic storms at these latitudes. Namely, the geomagnetic storms at polar region may be viewed as the results of a series of geomagnetic disturbances, that consist of a series of pulses (seen in the $A E$ index), each lasting a few hours.

\section{Cross-correlations for HSS/CIRs}

A complete time-series analysis of the HSS/CIR-driven geomagnetic activity was performed in Papers I and II. Here, we focus on the peak values of HSS/CIR solar-wind parameters and geomagnetic indices, to compare the ICME- and HSS/CIR-driven geomagnetic activity at various latitudes (Sect. 6).
Table 4. Correlation coefficients for the relationship between the HSS/CIR parameters and geomagnetic indices.

\begin{tabular}{lcccccc}
\hline \hline & \multicolumn{2}{c}{$A p$} & \multicolumn{2}{c}{$D s t$} & \multicolumn{2}{c}{$A E$} \\
& $R_{\text {lin }}$ & $R_{\text {pow }}$ & $R_{\text {lin }}$ & $R_{\text {pow }}$ & $R_{\text {lin }}$ & $R_{\text {pow }}$ \\
\hline$B$ & 0.43 & 0.56 & -0.70 & -0.72 & 0.43 & 0.51 \\
$V$ & 0.16 & 0.18 & -0.04 & -0.02 & 0.15 & 0.16 \\
$B V$ & 0.56 & 0.62 & -0.64 & -0.67 & 0.50 & 0.58 \\
\hline
\end{tabular}

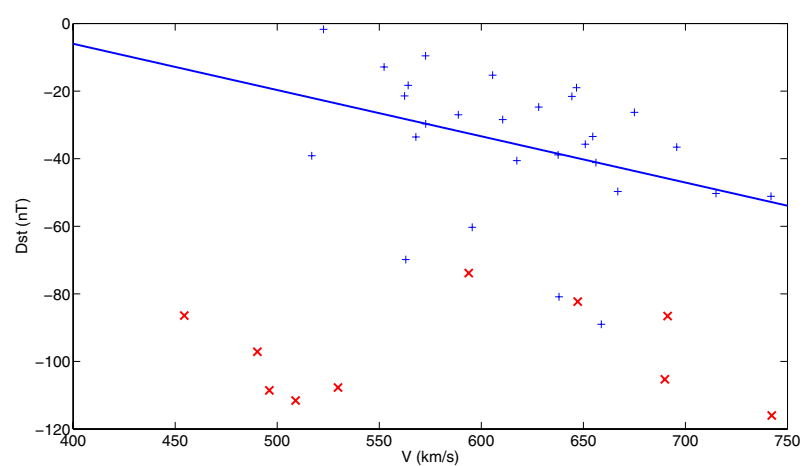

Fig. 5. Linear least-squares fit $V-D s t$ for HSS/CIRs. The events from Paper I and Paper II are depicted by the blue pluses (the corresponding linear fit $D s t=-0.14 V+49$ is shown by blue line), and the events from Zhang et al. (2007a) by red crosses.

\subsection{Cross-correlation between HSS/CIR parameters and geomagnetic indices}

In the following we investigate the relationship between the HSS/CIR parameters $B, V$, and $B V$, and geomagnetic indices $A p, D s t$, and $A E$, by applying the cross-correlation analysis. As in the case of ICMEs, for all relationships we apply linear and power-law least-squares fitting.

In Table 4 we present the correlation coefficients of the linear and power-law fit. The table shows that there is no correlation between geomagnetic indices and $V$. On the other hand, the correlations between geomagnetic indices and $B$ and $B V$ are relatively well defined. The best ones are $B-D s t$ and $B V-D s t$ correlations. The $B-A p$ and $B-A E$ correlations are somewhat weaker, while $B V-A p$ correlation is somewhat better than the $B V-A E$ correlation. For all dependencies, a power law is found to better describe the data than the linear fit.

Regarding the relationship between $V$ and geomagnetic indices, it is interesting to note that when we examine separately events from our previous papers (Paper I and Paper II), namely CIR/HSSs occurring in 2005 and 2006, which were not affected by ICMEs, we obtain moderate-to-weak correlations for $V-A p$ $(R=0.42)$ and $V-D s t(R=-0.37)$, and somewhat better correlation for $V-A E(R=0.58)$. As an illustration, we show in Fig. 5 the scatter plot $V-D s t$. The linear fit for the data from Paper I and Paper II (displayed as blue plusses) is shown by a blue line. Inspecting the plot, three group of events can be noticed: 1) the events in the lower-left part having $450<V<550 \mathrm{~km} \mathrm{~s}^{-1}$ and $-120<D s t<-80 \mathrm{nT}$ (events only from Zhang et al. 2007a); 2) the events in the range $550<V<750 \mathrm{~km} \mathrm{~s}^{-1}$ and $-120<D s t<-60 \mathrm{nT}$ (events from Zhang et al. 2007a and from our Paper I and Paper II) and 3) the events in the uppermost part having $530<V<750 \mathrm{~km} \mathrm{~s}^{-1}$ and $-50<D s t<0$ nT (events only from Paper I and Paper II).

The events from Zhang et al. (2007a) were selected as having intense storms. Hence, they will lie at lower Dst than the other set of events. We note that these strongest CIR-related events 

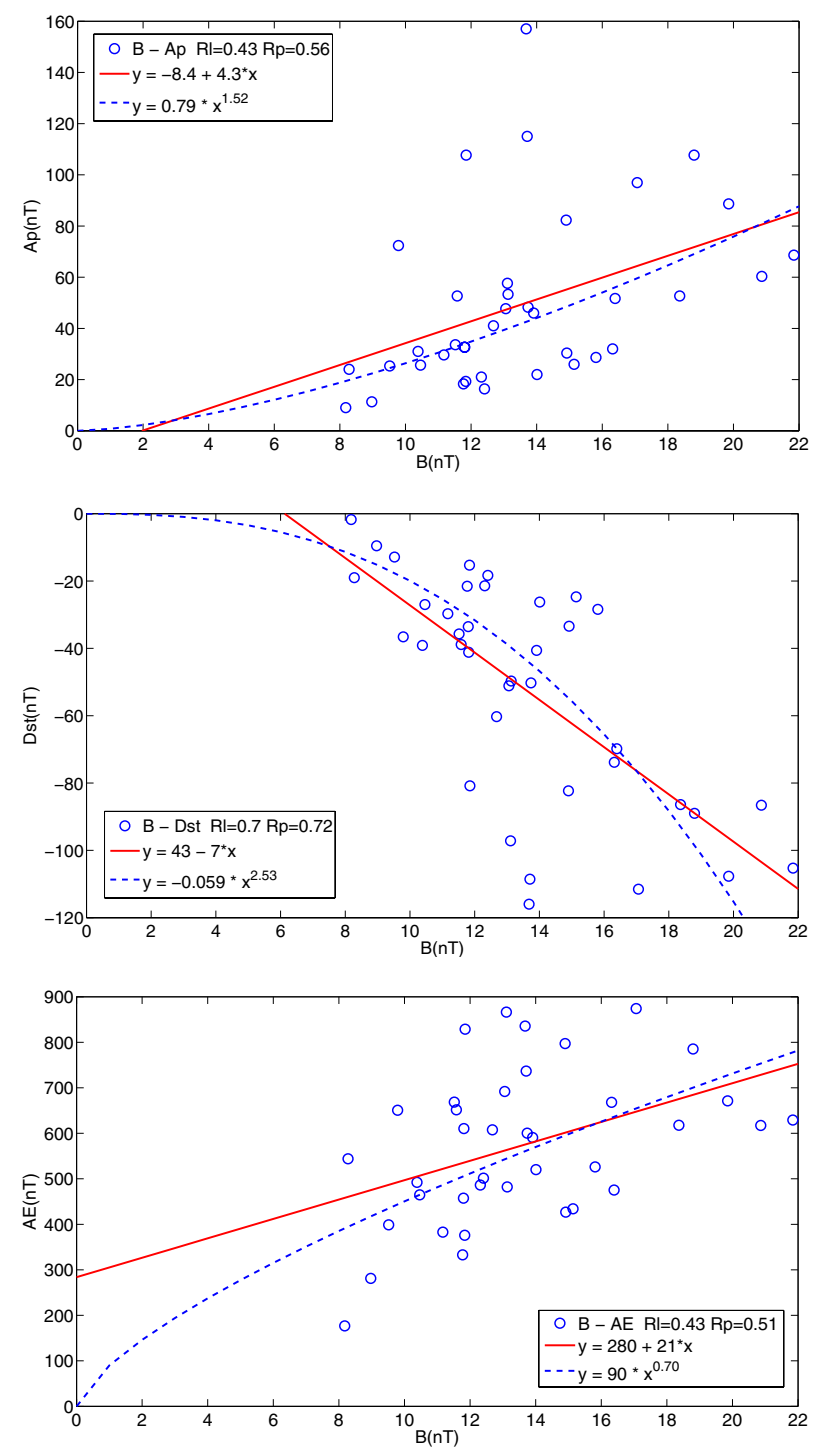

Fig. 6. Linear and power-law least-squares fits $B-A p, B-D s t$, and $B-A E$ for HSS/CIRs. Fit parameters and the correlation coefficients are presented in the insets.

are distributed through different years and phases of the solar cycle, when the general state of the heliosphere is significantly more complex than it is in the phases when the occurrence rate of CMEs is low. One may speculate that because of the permanent CMEs activity, these CIRs (from Zhang et al. 2007a) produced different effects than those that occurred in the period of low CME activity (from Paper I and Paper II).

The scatter plots $B-A p, B-A E$, and $B-D s t$ displayed in Fig. 6 show that $B$ has to reach $8 \mathrm{nT}$ in order to trigger at least some geomagnetic activity. It can also be noticed that there is an upper limit in $D s t$ and lower limit in $A p$ data distributions that roughly have a form of a power-law. The largest $D s t$ dip $(-116 \mathrm{nT})$ and the highest $A p$ peak $(157 \mathrm{nT})$ occur at $\sim 14 \mathrm{nT}$. These values are about two (four) times higher than the $D s t$ $(A p)$ values given by the corresponding linear-regression fits. At this value of $B$, the upper (lower) limits suggest $D s t \sim-20 \mathrm{nT}$ $(A p \sim 20 \mathrm{nT})$. We note that the power-law fit is more pronounced for $B-A p$ dependence. The $B-A E$ data distribution reveals the presence of both the lower and upper limit that have the form of a power-law curve; as soon as $B$ gets enhanced, it results in a recognizable $A E$ disturbance.
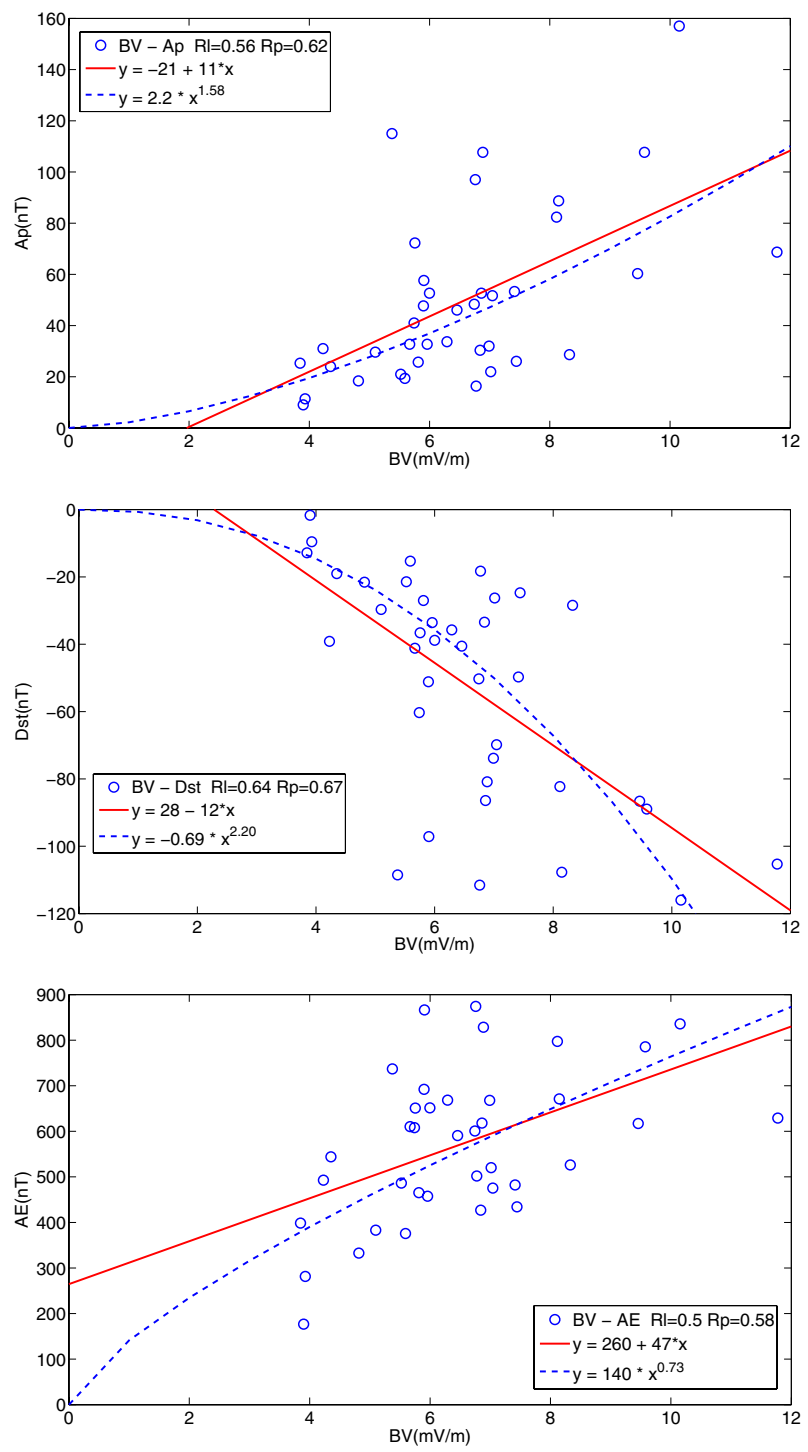

Fig. 7. Linear and power-law least-squares fit $B V-A p, B V-D s t$ and $B V-A E$ for HSS/CIRs. Fit parameters and the correlation coefficients are presented in the insets.

The $B V-A p, B V-A E$, and $B V-D$ st data are drawn in Fig. 7 and show that $B V$ has to reach $\sim 4 \mathrm{mV} \mathrm{m}^{-1}$ in order to trigger at least some geomagnetic activity. Generally, stronger geomagnetic activity is related to higher $B V$ values. The highest values of $A p(157 \mathrm{nT})$ and Dst $(-116 \mathrm{nT})$ are associated with the strongest $B V\left(10 \mathrm{mV} \mathrm{m}^{-1}\right)$ while the strongest $A E(874 \mathrm{nT})$ is associated with $B V=7 \mathrm{mV} \mathrm{m}^{-1}$. Interestingly, we obtain $B V-D s t$ dependence that has a form close to the quadratic (the powerlaw exponent is 2.2). Furthermore, the power-law fit indicates that, on average, HSS/CIRs drive intense low-latitude geomagnetic storms $(D s t<-100)$ for $B V$ above $9 \mathrm{mV} \mathrm{m}^{-1}$. However, according to the data scatter, such storms can already occur at $B V \sim 5 \mathrm{mV} \mathrm{m}^{-1}$.

Finally, we note that $A p$ and $A E$ correlations with $B V$ are better than with $B$, although the correlations with $V$ is practically zero. Namely, the velocity here plays the important role only indirectly. Higher velocities lead to a stronger compression, so the $B$ enhancement is stronger, and thus, $B V$ gets stronger too. For $D s t$, we found that $D s t-B V$ correlations are weaker than the $D s t-B$ correlation, suggesting that $V$ does not play an important role in producing $D$ st. 
Table 5. Linear least-squares correlation coefficients for the relationship between geomagnetic indices.

\begin{tabular}{lcc}
\hline \hline & ICMEs & HSS/CIRs \\
\hline$A p-D s t$ & -0.92 & -0.85 \\
$A E-D s t$ & -0.67 & -0.81 \\
$A E-A p$ & 0.76 & 0.79 \\
\hline
\end{tabular}

\section{Comparison of ICME and $\mathrm{HSS} / \mathrm{CIR}$ cross-correlation results}

\subsection{Cross-correlation between geomagnetic indices}

In Table 5, the correlation coefficients for the linear relationships between geomagnetic indices for ICMEs and HSS/CIRs are compared. The presented values show that in the case of ICMEs the best correlation is $D s t-A p$, and the lowest is $D s t-A E$. For HSS/CIRs, again the strongest correlation is $D s t-A p$. We note that the $D s t-A p$ correlation is better for ICMEs than for HSS/CIRs, while both $D s t-A E$ and $A E-A p$ correlations are higher for HSS/CIRs. This is consistent with the locations of the geomagnetic observatories used for deriving a certain geomagnetic index. The obtained correlations indicate that for stronger storms (ICMEs), the auroral oval shifts to lower latitudes and the substorm strength, as quantified by the $A E$ index, starts showing a reduced level of activity.

\subsection{Relationship between solar wind parameters and geomagnetic indices}

By comparing Tables 2 and 4 one finds that the corresponding correlations are weaker for HSS/CIRs than for ICMEs. In particular, the correlations between all three geomagnetic indices and $V$ are significant for ICMEs, while no correlations is found for HSS/CIRs.

In the following, we compare in detail the ICMEs and HSS/CIRs linear dependencies $B V-A p, B V-A E$, and $B V-D s t$. To compare the significance of these relationships (as quantified by the correlation coefficients), it is necessary to use the same dynamical $B V$ range, for both ICMEs and HSS/CIRs. Thus, we consider here the ICMEs for which $B V<12 \mathrm{mV} \mathrm{m}^{-1}$, which is the highest value of $B V$ in the studied HSS/CIR sample. The outcome of the cross-correlation analysis is presented in Table 6. As expected, the ICME correlations in the restricted range of $B V$ values are weaker than the ICME correlations for the whole $B V$ range. Now, only for $A p$ is the ICME correlation coefficient considerably higher than for HSS/CIRs $(R=0.79$ and $R=0.56$, respectively); the correlations for the $D s t$ and $A p$ indices are almost the same. Table 6 reveals that the slopes of the regression lines for ICMEs and HSS/CIRs are the same within the error limits for all three indices. The parameter $b$ of the linear leastsquares fits for HSS/CIRs are lower, meaning that at the same $B V$ ICMEs are more geoeffective at all latitudes. In the considered range, the mean $B V$ value is $7.4 \pm 2.5 \mathrm{mV} \mathrm{m}^{-1}$ for ICMEs and $6.5 \pm 1.7 \mathrm{mV} \mathrm{m}^{-1}$ for HSS/CIRs. In case of ICMEs, the mean values of $A p, D s t$, and $A E$ are $63 \pm 47 \mathrm{nT},-73 \pm 45 \mathrm{nT}$, and $652 \pm 234 \mathrm{nT}$, respectively. For HSS/CIRs these values are somewhat lower, equaling $49 \pm 33 \mathrm{nT},-52 \pm 33 \mathrm{nT}$, and $572 \pm 165 \mathrm{nT}$.

\section{Relationship to the coronagraphic CME speed}

For space-weather forecasting purposes, in the following we apply the cross-correlation analysis to the relationships between:

- peak values of the ICME parameters $B$ and $V$, and the coronagraphic $\mathrm{CME}$ velocity $V_{\mathrm{CME}}$;
Table 6. Linear least-squares fits $(y=a x+b)$ and correlation coefficients $R$ describing the relationships between $B V\left(B V<12 \mathrm{mV} \mathrm{m}^{-1}\right)$ and geomagnetic indices.

\begin{tabular}{lcccccc}
\hline \hline & $a_{\mathrm{ICME}}$ & $a_{\mathrm{CIR}}$ & $b_{\mathrm{ICME}}$ & $b_{\mathrm{CIR}}$ & $R_{\mathrm{ICME}}$ & $R_{\mathrm{CIR}}$ \\
\hline$D s t$ & $12 \pm 2$ & $12 \pm 2$ & $13 \pm 19$ & $28 \pm 17$ & -0.65 & -0.64 \\
$A p$ & $14 \pm 2$ & $11 \pm 3$ & $-35 \pm 14$ & $-21 \pm 18$ & 0.79 & 0.56 \\
$A E$ & $45 \pm 13$ & $47 \pm 14$ & $328 \pm 99$ & $265 \pm 93$ & 0.52 & 0.50 \\
\hline
\end{tabular}

Notes. Subscripts ICME and CIR refer to ICMEs and HSS/CIRs, respectively.
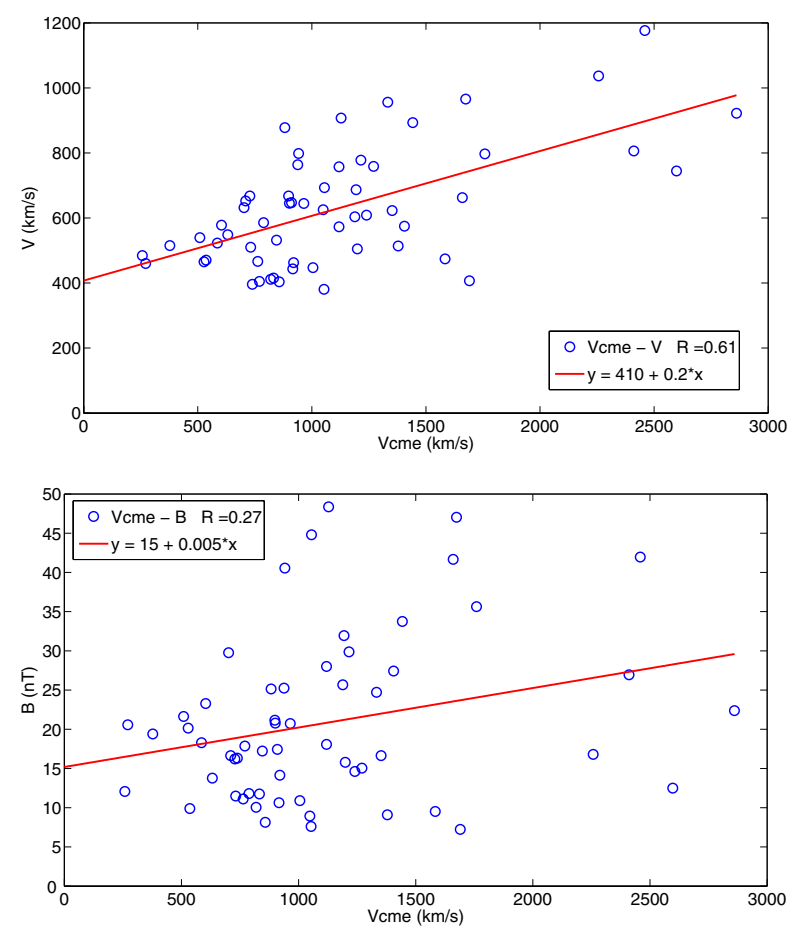

Fig. 8. Dependence of ICME peak velocity $V(t o p)$ and ICME peak magnetic field $B$ (bottom) on the coronagraphic CME mean speed $V_{\mathrm{CME}}$. The linear least-squares fits are shown by the solid lines (parameters and the correlation coefficients $R$ are shown in the insets).

- peak values of geomagnetic indices $A p, D s t$, and $A E$ and the velocity $V_{\mathrm{CME}}$.

In Fig. 8 we show how the ICME parameters $V$ and $B$ are related to the mean speed of CMEs measured in the LASCO field of view, $V_{\mathrm{CME}}$. The ICME speed $V$ is relatively well correlated with $V_{\mathrm{CME}}(R=0.61)$, whereas the correlation $V_{\mathrm{CME}}-B$ is rather weak $(R=0.27)$. Values of $V_{\mathrm{CME}}$ are in the range $\sim 250-3000 \mathrm{~km} \mathrm{~s}^{-1}$. The corresponding speeds at the Earth (1 AU) are in the range $\sim 380$ to $\sim 1200 \mathrm{~km} \mathrm{~s}^{-1}$. The narrower range of speeds at Earth is a direct result of the interaction between CMEs and the solar wind (e.g., Lindsay et al. 1999; Gopalswamy 2010; Vršnak et al. 2010).

The $V\left(V_{\mathrm{CME}}\right)$ linear fit gives the value $V=410 \mathrm{~km} \mathrm{~s}^{-1}$ for the $y$-axis intercept $\left(V_{\mathrm{CME}}=0\right)$. Furthermore, we find that $V$ becomes equal to $V_{\mathrm{CME}}$ for $V_{\mathrm{CME}}=512 \mathrm{~km} \mathrm{~s}^{-1}$. Thus, for $V_{\mathrm{CME}}$ below this value, ICMEs are accelerated, i.e., $V>V_{\mathrm{CME}}$, whereas if $V_{\mathrm{CME}}$ is higher than this value, ICMEs show $V<V_{\mathrm{CME}}$, implying they are decelerated.

Although the correlation $V_{\mathrm{CME}}-B$ is quite weak, the linear fit (Fig. 8, bottom panel) indicates that higher-speed CMEs tend to have a stronger magnetic field $B$ near the Earth. However, we note that the fastest two CMEs in our sample were associated with only modestly enhanced $B$ at the Earth. We note also that 

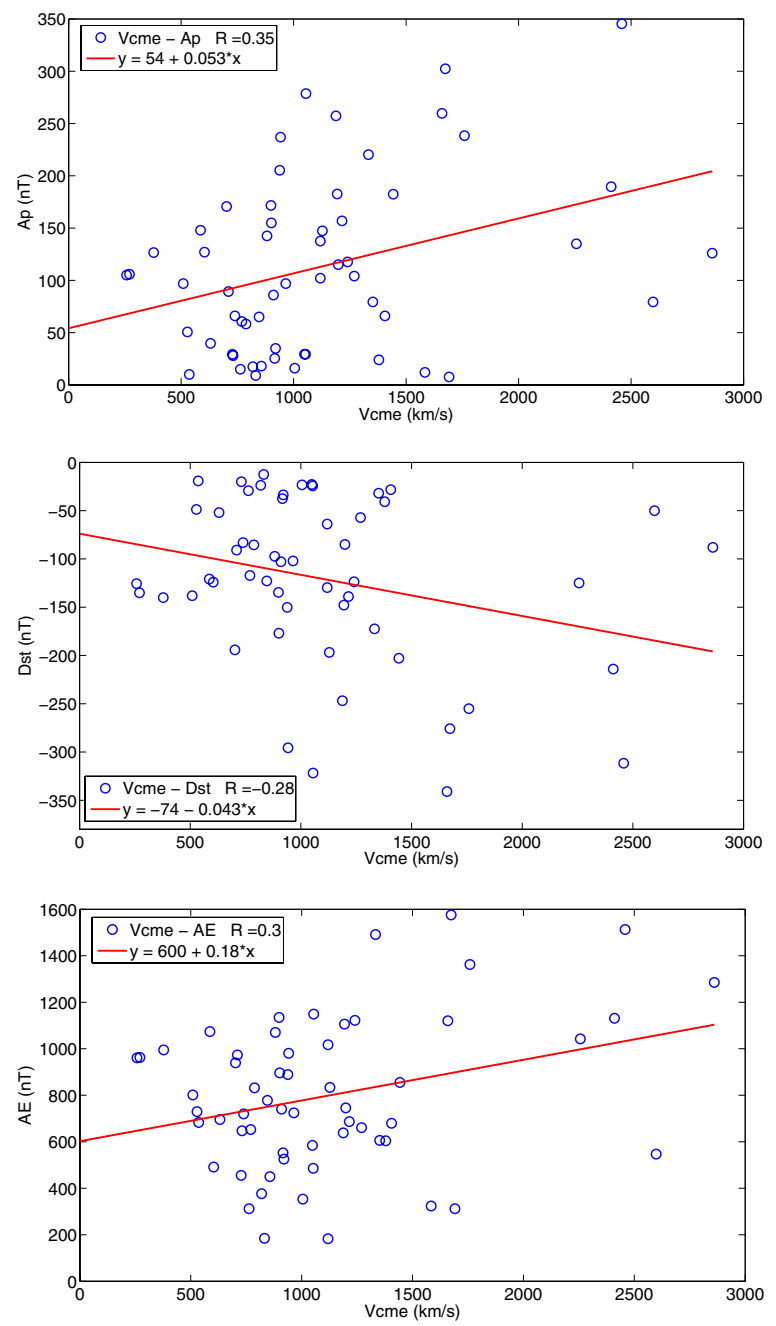

Fig. 9. Correlations $V_{\mathrm{CME}}-A p, V_{\mathrm{CME}}-D s t$, and $V_{\mathrm{CME}}-A E$.

slow CMEs do not result in a strong ICME field (data void in the upper-left part of the graph). The relationship between ICME parameters $B, V$, and $\mathrm{CME}$ speed have also been discussed by, e.g., Lindsay et al. (1999); Gopalswamy $(2008,2010)$ and Richardson \& Cane (2011).

In Fig. 9 we present the relationships between $V_{\mathrm{CME}}$ and $A p, D s t$, and $A E$. The correlation coefficients are considerably lower (by about a factor of 2) than for the correlations obtained employing the ICME speed $V$. The best correlation is found for the dependence $V_{\mathrm{CME}}-A p(R=0.35)$. The correlation coefficients for the relationships $V_{\mathrm{CME}}-A E$ and $V_{\mathrm{CME}}-D s t$ are similar ( $R=0.30$ and $R=-0.28$, respectively),

Finally, it should be noted that there are fast CMEs that have almost no geomagnetic effect at low and mid latitudes as seen from the $V_{\mathrm{CME}}-A p$ and $V_{\mathrm{CME}}-D s t$ graphs. On the other hand, in the case of $A E$, the lower limit shows that at large $V_{\mathrm{CME}}$ there will be always some geomagnetic activity triggered.

\section{Discussion and conclusions}

The geomagnetic activity associated with 63 ICMEs and 38 HSS/CIRs in the period 1998-2006 has been studied. We considered the effect of the overall ICME disturbances, which includes the shock, the sheath region, and the magnetic ejection, making no distinction between the geoeffectiveness of individual structural elements. Most of the selected HSS/CIRs occurred in periods when the activity of coronal mass ejections was low (two periods in 2005 and 2006 were considered, as in Paper II).
We have analyzed various linear and power-law least-squares relationships, separately for ICMEs and HSS/CIRs, to examine similarities/differences in the magnetospheric response at low, mid, and high latitudes to ICMEs and HSS/CIRs. We summarize our results as follows (the quoted values of $V, B, D s t, A p$, and $A E$ represent six-hours averages).

For ICMEs the fitted linear relation $V-B$ clearly shows a trend that ICMEs with stronger magnetic field propagate at higher velocities. This has implications for geoeffectiveness since the product $B V$, related to the electric field, is enhanced by both factors, $V$ and $B$.

For ICMEs the power-law least-squares fits are found to be important only for the relationships between geomagnetic indices and $B V$. The performed analysis for two time resolutions obtained by one-hour (three-hour for $A p$ ) and six-hour averaging show: Dst is linearly dependent on $B V$, and does not depend on the data resolution. Below $\sim 5 \mathrm{mV} \mathrm{m}^{-1}$, all Dst peaks are below $-50 \mathrm{nT}$. The fits indicate that an intense geomagnetic storm (Dst $<-100 \mathrm{nT})$ and a very intense storm $(D s t<-300 \mathrm{nT})$ occur, on average, at $B V \sim 10 \mathrm{mV} \mathrm{m}^{-1}$ and $\sim 35 \mathrm{mV} \mathrm{m}^{-1}$, respectively. However, the data distribution shows that such storm intensities could occur at $B V$ even below these values. For the $B V-A p$ relationship, the power-law dependence is slightly better than the linear dependence at both resolutions. Below $B V \sim 5 \mathrm{mV} \mathrm{m}^{-1}, A p$ peaks have low values $(<50 \mathrm{nT})$. The cross-correlation coefficient of the power-law $B V-A p$ fit is the largest one. In the case of the $B V-A E$ relationship, the linear and power-law fits are very similar at the six-hour resolution, while at the one-hour resolution the power-law provides a better fit. This indicates that the response of the polar magnetosphere is more sensitive to fast fluctuations in the solar wind (here quantified by the product $B V$ ) than is the equatorial current system. Thus, for studying the nature of the temporal variability of the magnetic activity at high latitudes that is caused by ICMEs, the one-hour resolution is probably more appropriate. The powerlaw exponent is close to $1 / 2$, regardless of the resolution. The $B V-A E$ data distribution indicates a pronounced upper limit to the data. This upper-limit boundary, located about $500 \mathrm{nT}$ above the power-law fit, rises up to $B V \sim 25 \mathrm{mV} \mathrm{m}^{-1}$, and then indicates a kind of saturation. On the other hand, at very high $B V$ values, there is also a lower limit to the data, i.e., there is no $A E$ below $\sim 600 \mathrm{nT}$. It is worth noting that even at very low $B V$ peak values $\left(\$ 5 \mathrm{mV} \mathrm{m}^{-1}\right)$, some significant $A E$ values are observed, up to $700 \mathrm{nT}$.

For HSS/CIRs there is no correlation between geomagnetic indices and $V$. However, when we examine separately events from our previous papers (Paper I and Paper II), namely CIR/HSSs occurring in 2005 and 2006, which were not affected by ICMEs, we obtain moderate-to-weak correlations between $V$ and geomagnetic indices.

For all HSS/CIRs dependencies, power law is found to describe the data better than the linear least-squares fit. The best correlations are $B-D s t$ and $B V-D$ st.

The HSS/CIRs $B V-A p, B V-A E$, and $B V-D s t$ data distribution show that $B V$ has to reach $\sim 4 \mathrm{mV} \mathrm{m}^{-1}$ in order to drive at least some geomagnetic activity.

The power-law $B V-D s t$ fit indicates that, on average, HSS/CIRs drive intense low-latitude geomagnetic storms $(D s t<-100)$ for $B V$ above $9 \mathrm{mV} \mathrm{m}^{-1}$. However, according to the data scatter, these storms can occur already at $B V \sim 5 \mathrm{mV} \mathrm{m}^{-1}$.

We observed that the HSS/CIRs velocity is important for $A p$ and $A E$, though only indirectly, while it does not play an important role in producing $D$ st. 
The $D s t-A p$ correlation is better for ICMEs than for HSS/CIRs, while both $D s t-A E$ and $A E-A p$ correlations are higher for HSS/CIRs.

Within the same dynamical $B V$ range $\left(B V<12 \mathrm{mV} \mathrm{m}^{-1}\right)$, only the ICMEs $A p(B V)$ correlation is considerably stronger than the HSS/CIRs $A p(B V)$ correlation $(R=0.79$ and $R=0.56$, respectively). Nevertheless, the regression parameters indicate that at the same value of $B V$ ICMEs are more geoeffective at all latitudes.

For ICMEs the correlation coefficients for $A E(B V)$ and $\operatorname{Dst}(B V)$ drop significantly when $B V<12 \mathrm{mV} \mathrm{m}^{-1}$ was analyzed. This may indicate that both the polar electrojet and ring current are quite sensitive to the $B V$ amplitude.

The ICME speed $V$ is relatively well correlated with CME speed $V_{\mathrm{CME}}(R=0.61)$, whereas the correlation $V_{\mathrm{CME}}-B$ is rather weak $(R=0.27)$. Anyway, the linear fit $V_{\mathrm{CME}}-B$ indicates that higher-speed CMEs tend to have a stronger magnetic field $B$ near the Earth. We note also that slow CMEs do not result in a strong ICME field.

We observed that there are fast CMEs that have almost no geomagnetic effect at low and mid latitudes. On the other hand, at large $V_{\mathrm{CME}}$ there will be always some geomagnetic activity triggered at high latitudes. This is an important finding regarding the space weather forecasting.

For both ICMEs and HSS/CIRs, the maximum peak values of different geomagnetic indices do not occur in the same event. This suggests that different current systems within the Earth's magnetosphere (as measured by ground magnetometers at different latitudes and quantified by geomagnetic indices) show different responses to the geoeffective solar wind flow.

The results clearly indicate different magnetospheric responses to the same input from the solar wind driver $(B V)$ at various latitudes. Furthermore, we observed relative differences in the response of different current systems within the Earth's magnetosphere to the impact of ICMEs and HSS/CIRs.

The ICME-driven activity shows that the development of the equatorial and polar current systems are not always in constant proportion (as we noted by Gonzalez et al. 1994). Namely, the $\operatorname{Dst}(B V)$ correlations are stronger than $A E(B V)$ correlations, and the $A E(D s t)$ dependence is the weakest one of the three geomagnetic indices analyzed. For ICMEs the highest impact at mid latitudes probably reflects the contribution from both current systems in these regions, and consequently a growth of ionospheric currents. Moreover, during the intense particles injection into the magnetosphere, the substorm currents may be formed at lower latitudes, and so will not be as strong as expected at high-latitude observatories. Instead, the $A p$ index will be additionally increased.

For HSS/CIRs, the $A E$ and $D s t$ are tightly related, suggesting that development of the ring current and auroral electrojet are closely related for this type of solar wind disturbances. This aspect should be investigated further.

In conclusion, our study shows that the magnetosphere reaction on both solar drivers (ICMEs and HSS/CIRs) is different at various latitudes. Thus, the full picture of the ICMEs and HSS/CIRs driven geomagnetic activities can be obtained only by studying the development of different current systems within the Earth's magnetosphere and ionosphere.

Acknowledgements. The presented work has received funding from the European Union Seventh Framework Programme (FP7/2007-2013) under grant agreement n 263252 [COMESEP].

\section{References}

Alves, M. V., Echer, E., \& Gonzalez, W. D. 2006, J. Geophys. Res. (Space Phys.), 111, A07S05

Bakare, N. O., \& Chukwuma, V. U. 2010, Indian J. Radio Space Phys., 39, 150 Borovsky, J. E., \& Denton, M. 2006, J. Geophys. Res., 111

Brueckner, G. E., Howard, R. A., Koomen, M. J., et al. 1995, Sol. Phys., 162, 357

Burlaga, L. F., \& Lepping, R. 1977, Planet. Space Sci., 25, 1151

Dal Lago, A., Gonzalez, W. D., de Gonzalez, A. L. C., \& Vieira, L. E. A. 2001, J. Atmos. Sol. Terr. Phys., 63, 451

Dumbović, M., Vršnak, B., Čalogović, J., \& Karlica, M. 2011, A\&A, 531, A91

Dungey, J. 1961, Phys. Rev. Lett., 6, 47

Echer, E., Gonzalez, W. D., Tsurutani, B., \& Gonzalez, A. 2008, J. Geophys. Res., 113, A05221

Georgieva, K., Kirov, B., \& Gavruseva, E. 2006, Phys. Chem. Earth, 31, 81

Gonzalez, W. D., \& Tsurutani, B. T. 1987, Planet. Space Sci., 35, 1101

Gonzalez, W. D., Joselyn, J., Kamide, Y., et al. 1994, J. Geophys. Res., 99, 5771

Gonzalez, W. D., Chía de Gonzalez, A. L., Dal Lago, A., et al. 1998, Geophys. Res. Lett., 25, 963

Gopalswamy, N. 2008, J. Atmos. Sol. Terr. Phys., 70, 2078

Gopalswamy, N. 2010, in IAU Symp., 264, 326

Gopalswamy, N., Akiyama, S., Yashiro, S., Michalek, G., \& Lepping, R. P. 2008, J. Atmos. Sol. Terr. Phys., 70, 245

Gosling, J. T. 1996, ARA\&A, 34, 35

Huttunen, K. E. J., Schwenn, R., Bothmer, V., \& Koskinen, H. E. J. 2005, Ann. Geophys., 23, 625

Ji, E., Moon, Y., Kim, H., \& Lee, H. 2010, J. Geophys. Res., 115, A10232

Kane, R. P. 2005, J. Geophys. Res. (Space Phys.), 110, A9, A02213

King, H. J. 1986, in Solar Wind-Magnetosphere Coupling, eds. Y. Kamide, \& J. Slavin (Tokyo: Terra Scientific), 163

Kivelson, M. G., \& Russel, C. T. 1995, Introduction to Space Physics (Cambridge University press)

Lavraud, B., Thomsen, M. F., Borovsky, J. E., Denton, M. H., \& Pulkkinen, T. I. 2006, J. Geophys. Res. (Space Phys.), 111, A09208

Lindsay, G. M., Luhmann, J. G., Russell, C. T., \& Gosling, J. T. 1999, J. Geophys. Res., 104, 12515

McComas, D., Bame, S. J., Barker, P., et al. 1998, Space Sci. Rev., 86, 563

Möstl, C., Farrugia, C. J., Kilpua, E. K. J., et al. 2012, ApJ, 758, 10

Owens, M., Cargill, P., Pagel, C., Siscoe, G., \& Crooker, N. 2005, J. Geophys. Res., 110, A0110

Prolss, G. 2004, Physics of the Earth's Space Environment (Berlin, Heidelberg: Springer-Verlag)

Richardson, I. G., \& Cane, H. 2010, Sol. Phys, 264, 189

Richardson, I. G., \& Cane, H. 2011, Space Weather, 9, S07005

Richardson, I. G., \& Cane, H. 2012, J. Space Weather Space Climate, 2, A01

Richardson, I. G., Cliver, E., \& Cane, H. 2001, Geophys. Res. Lett., 28, 2569

Richardson, I. G., Webb, D. F., Zhang, J., et al. 2006, J. Geophys. Res., 111, A07S09

Schwenn, R. 1983, Space Sci. Rev., 34, 85

Schwenn, R., dal Lago, A., Huttunen, E., \& Gonzalez, W. D. 2005, Ann. Geophys., 23, 1033

Smith, C. W., L'Heureux, J., Ness, N. F., et al. 1998, Space Sci. Rev., 86, 613

Stone, E., Frandsen, A., Mewaldt, R., et al. 1998, Space Sci. Rev., 86, 1

Sugiura, M., \& Chapman, S. 1960, Abandal. Akad. Wiss., Gottingen Math. Phys., K1, 4

Tsurutani, B. T., \& Gonzalez, W. D. 1987, Planet. Space Sci., 35, 405

Tsurutani, B., \& Gonzalez, W. 1997, in The interplanetary causes of magnetic storms: A review, eds. B. Tsurutani, W. Gonzalez, Y. Kamide, \& J. Arballo, Geophys. Monogr. Ser., 98, 1

Tsurutani, B. T., Gonzalez, W. D., Guarnieri, F., et al. 2004, J. Atmos. Sol. Terr. Phys., 66, 167

Tsurutani, B. T., McPherron, R. L., Gonzalez, W. D., et al. 2006, J. Geophys. Res., 111,1

Verbanac, G., Lühr, H., Rother, M., Korte, M., \& Mandea, M. 2007, Earth, Planet. Space, 59, 251

Verbanac, G., Vršnak, B., Temmer, M., Mandea, M., \& Korte, M. 2010, J. Atmos. Sol. Terr. Phys., 607

Verbanac, G., Vršnak, B., Temmer, M., \& Veronig, A. 2011a, A\&A, 526, A20

Verbanac, G., Vršnak, B., Živković, S., et al. 2011b, A\&A, 533, A49

Vršnak, B., Temmer, M., \& Veronig, A. 2007, Sol. Phys., 240, 331

Vršnak, B., Žic, T., Falkenberg, T. V., et al. 2010, A\&A, 512, A43

Wang, Y., Shen, C. L., Wang, S., \& Ye, P. Z. 2003, Geophys. Res. Lett., 30, 2039

Yermolaev, Y., \& Yermolaev, M. 2006, Adv. Space Res, 37, 6, 1175

Zhang, J., Dere, K. P., Howard, R. A., \& Bothmer, V. 2003, ApJ, 582, 520

Zhang, J., Richardson, I. G., Webb, D. F., et al. 2007a, J. Geophys. Res. (Space Phys.), 112, 12103

Zhang, J., Richardson, I. G., Webb, D. F., et al. 2007b, J. Geophys. Res. (Space Phys.), 112, 10102 